\title{
Adaptive Optics Program Update at TMT
}

\author{
C. Boyer \\ TMT Observatory Corp., 100 W. Walnut St, Pasadena, CA, USA 91124;
}

\begin{abstract}
The TMT first light Adaptive Optics (AO) facility consists of the Narrow Field Infra-Red AO System (NFIRAOS), the associated Laser Guide Star Facility (LGSF) and the AO Executive Software (AOESW). NFIRAOS is a 60 x 60 laser guide star (LGS) multi-conjugate AO (MCAO) system, which provides uniform, diffraction-limited performance in the $\mathrm{J}, \mathrm{H}$, and $\mathrm{K}$ bands over 17-34 arc sec diameter fields with 50 per cent sky coverage at the galactic pole, as required to support the TMT science cases. NFIRAOS includes two deformable mirrors, six laser guide star wavefront sensors, one high order Pyramid WFS for natural guide star AO, up to three low-order, IR, natural guide star on-instrument wavefront sensors (OIWFS) within each client instrument, and up to four guide windows on the science detectors (ODGW). The first light LGSF system includes six sodium lasers required to generate the NFIRAOS laser guide stars.

In this paper, we will provide an update on the progress in designing, prototyping and fabricating the TMT first light AO systems and their components over the last two years. This will include the final design activities for NFIRAOS, preliminary design activities for the LGSF and AOESW, final design and prototyping activities for the deformable mirrors, final design and fabrication activities for the visible detectors, preliminary design and prototyping activities for the NFIRAOS visible cameras, and final design activities for the NFIRAOS Real Time Controller (RTC). Comprehensive and detailed $\mathrm{AO}$ modeling is also continuing to support the design and development of the first light $\mathrm{AO}$ facility.
\end{abstract}

Keywords: Adaptive optics program, extremely large telescopes

\section{INTRODUCTION}

The first light Adaptive Optics (AO) architecture for the TMT has been defined to provide near-diffraction-limited wavefront quality and high sky coverage in the near infra-red (IR) for the first TMT science instruments IRIS (InfraRed Imaging Spectrograph) ${ }^{[2]}$ and IRMS (InfraRed Multislit Spectrometer). It is a Laser Guide Star (LGS) Multi Conjugate AO (MCAO) architecture consisting of (i) the Narrow Field IR AO System (NFIRAOS), which feeds up to three science instrument ports after sensing and correcting for wavefront aberrations introduced by the atmospheric turbulence and the telescope itself, (ii) the Laser Guide Star Facility (LGSF), which generates multiple LGS in the mesospheric sodium layer with the brightness, beam quality and asterism geometry required by both NFIRAOS and later the second generation of TMT AO systems, and (iii) the Adaptive Optics Sequencer of the AO Executive Software, which automatically coordinates the operations of the AO systems with the remainder of the observatory for safe and efficient observations.

Significant progress has been made in designing, modeling and prototyping these systems and their AO components over the last two years. The NFIRAOS team is progressing full speed toward a final design review in June 2018. Several major NFIRAOS components, which have been sub-contracted with Canadian industry, have already completed their final design or are about to complete it. The NFIRAOS real time controller, also developed by the NFIRAOS team, is nearing its final design. The LGSF preliminary design work is progressing. Major simplifications of the LGSF top end have been performed to reduce complexity and improve access of the components. The interface between the LGSF and the telescope structure has been finalized.

*

cboyer@tmt.org; phone: 1626395 1623;www.tmt.org 
Some of the AO Executive Software components have successfully passed preliminary design. In the area of AO components, the deformable mirrors, the visible detectors and associated readout electronics have successfully completed their final designs. The visible detectors are being fabricated and prototypes of the visible detectors readout electronics are being tested. Prototype of the deformable mirrors are being fabricated and will be tested in the near future. Finally, TMT intends to select Toptica/MPBC lasers as its baseline for sodium lasers.

Extensive work is continuing to update and finalize the design requirement documents and interface control documents of the AO systems, $\mathrm{AO}$ components and their corresponding sub-systems, and to establish the traceability of these requirements. Finally, several AO modeling and analysis activities have been conducted in the areas of pyramid wavefront sensing, PSF reconstruction, detailing the LGS and NGS wavefront error budgets and adaptive secondary mirror performance, and to further support the development of requirements for the TMT AO systems and components.

\section{TMT FIRST LIGHT AO REQUIREMENTS AND ARCHITECTURE REVIEW}

The TMT top-level AO science requirements have been very stable for many years. They include high sky coverage at the galactic pole, diffraction limited performance in $\mathrm{J}, \mathrm{H}$ and $\mathrm{K}$ bands, high accuracy astrometry and photometry requirements, high optical throughput requirement and low background requirements. The TMT first light AO architecture has been developed to address these requirements and consists mainly of a Laser Guide Star (LGS) MultiConjugate AO (MCAO) system and a Laser Guide Star Facility (LGSF) to generate bright and efficient laser guide stars.

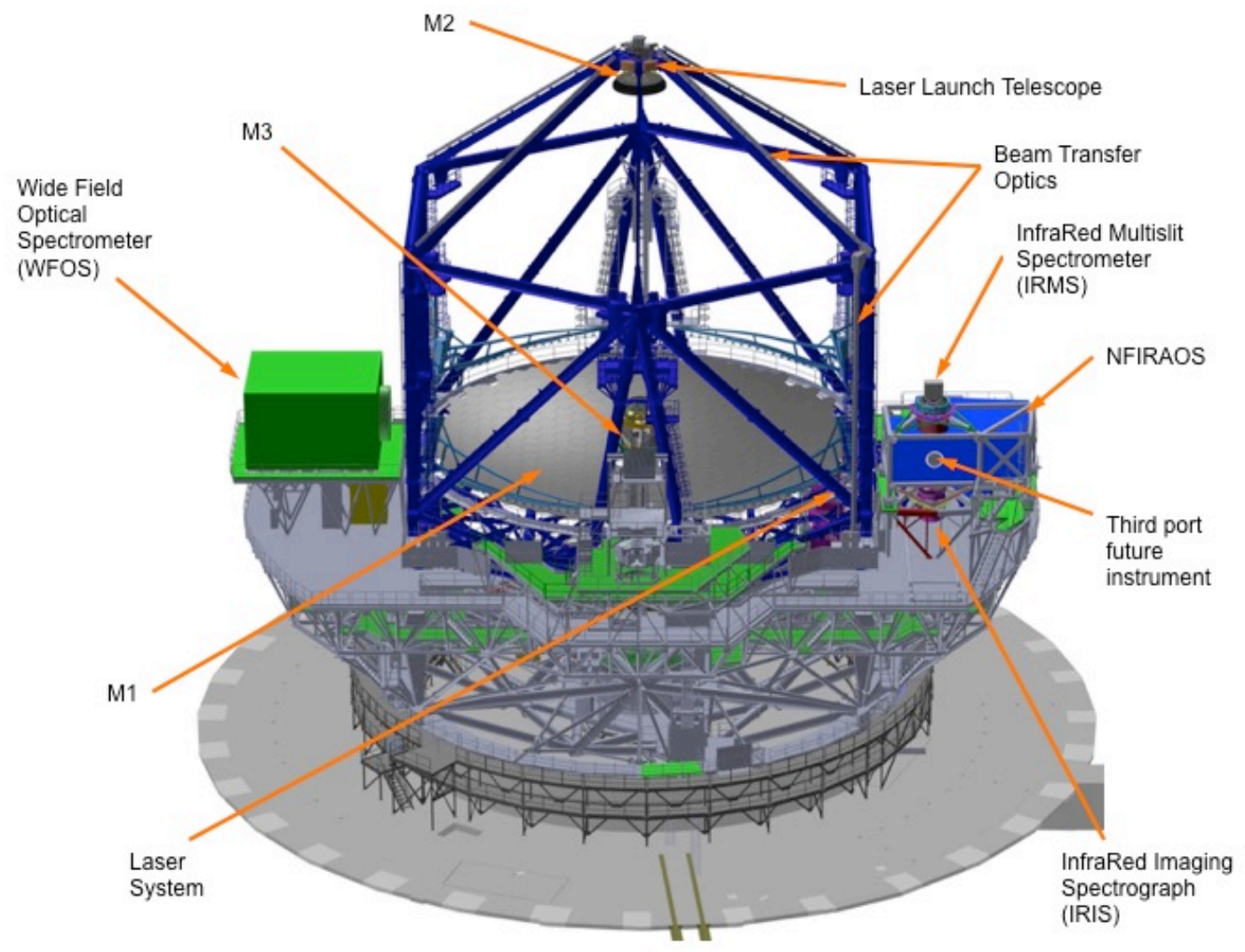

Figure 1: The TMT telescope with the first-light instruments and AO Systems (NFIRAOS and LGSF).

The first light AO architecture for TMT (Figure 1) consists of the following major systems:

- The LGS MCAO System, NFIRAOS, which is located on the TMT Nasmyth platform and relays light from the telescope to 3 science instrument ports after sensing and correcting for wavefront aberrations introduced by atmospheric turbulence and the observatory itself. NFIRAOS includes two DMs conjugated at $0 \mathrm{~km}(63 \times 63)$ and at $11.8 \mathrm{~km}(76 \times 76)$ with the DM conjugated to the ground mounted on a tip/tilt stage to reduce the number of 
optical surfaces. It also includes six 60x60 LGS WFS (one on-axis, and five in a pentagon with a radius of 35 arcsec), a $60 \times 60 \mathrm{NGS}$ WFS for operation without laser and operates at $800 \mathrm{~Hz}$. NFIRAOS is cooled at $-30^{\circ} \mathrm{C}$ to meet the low background requirement.

- The On-Instrument wavefront sensors (OIWFS) of the NFIRAOS instruments dedicated for tip/tilt/focus sensing in the near IR (IRIS provides three OIWFS and IRMS only one), and four On-Detector Guide Windows in IRIS serving as truth tip/tilt sensors.

- The LGSF generates multiple LGS in the mesospheric sodium layer with the brightness, beam quality, and asterism geometry required by both the first light AO system (NFIRAOS) and later the second generation of TMT AO systems. It includes: i) the lasers, which are attached to the inside of the $-X$ elevation journal, ii) the beam transfer optics optical path, which transports up to 9 laser beams in a square pattern along the telescope elevation structure to the telescope top end, iii) the LGSF top end, which formats and launches the laser asterisms (up to 4 different asterisms) to the sky from the laser launch telescope, and iv) the laser safety system.

- The Adaptive Optics Sequencer of the AO Executive Software, which automatically coordinates the operations of NFIRAOS, the OIWFS/ODGW and the LGSF with the remainder of the observatory for safe and efficient observations.

\section{FIRST LIGHT AO SYSTEM DESIGN PROGRESS}

\subsection{Narrow Field IR Adaptive Optics System (NFIRAOS)}

Since the AOELT4 Conference in 2015, the NFIRAOS team at NRC Herzberg in Victoria has continued to develop the NFIRAOS final design with the goal to have the Final Design Review in June $2018^{[3]}$.
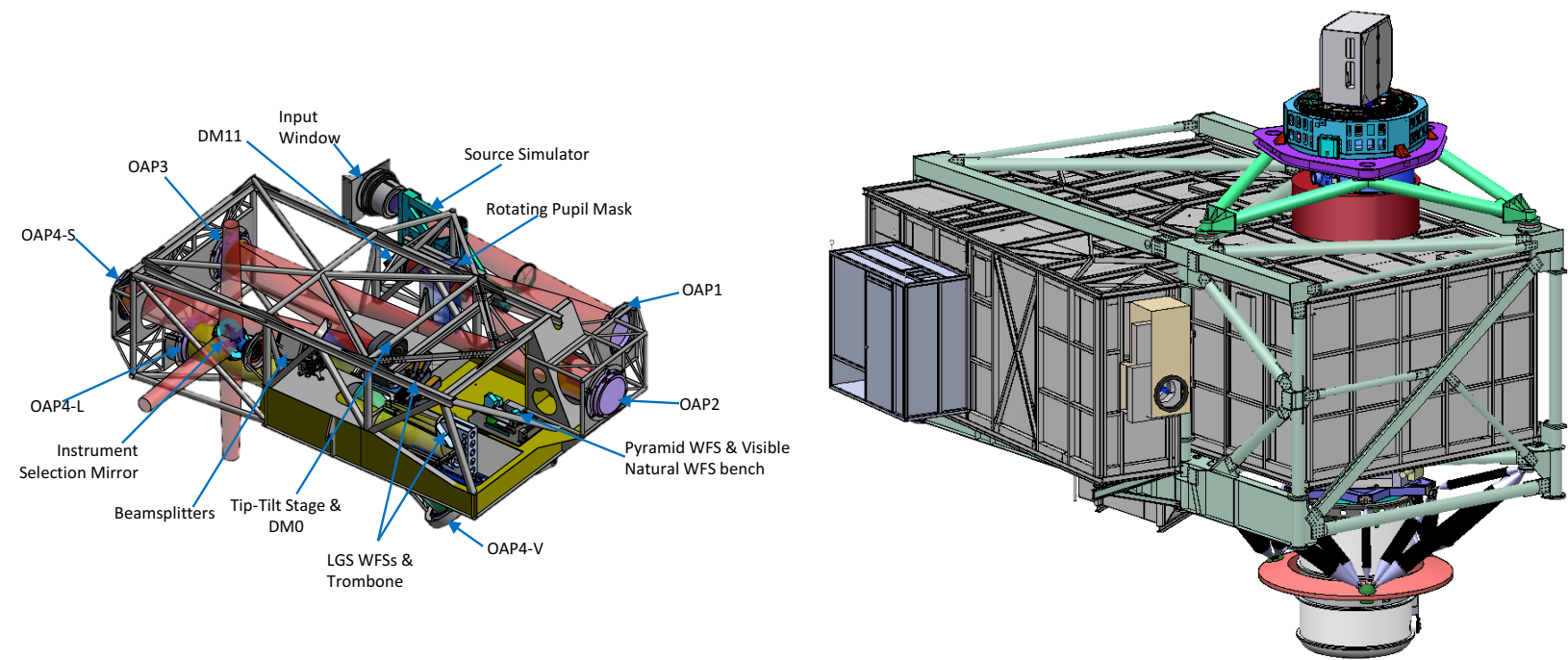

Figure 2: NFIRAOS optimized layout with the turbulence generator removed and the rotating pupil mask included (left). NFIRAOS optical enclosure with the IRIS instrument mounted at the bottom and the IRMS instrument mounted on the top (right).

The overall design, the system engineering activities, the design of the LGS wavefront system, the design of the software and electronics are developed in house. In parallel a total of 8 subcontracts has been awarded to Canada Industry to develop the final design of the main NFIRAOS components. Many components have already reached final design. In some cases, the cost for manufacturing and testing these components are high, and requires new trade off, descoping and, or optimization of the requirements.

- One example of these tradeoffs is the removal of the turbulence generator. The NFIRAOS turbulence generator consists of a turbulence screen conjugated at $+2 \mathrm{~km}$ and being deployed into the NFIRAOS common path (See Figure 2). When used in conjunction of artificially injected turbulence generated by corrupting the DM commands of both DMs, it is possible to emulate atmospheric turbulence consistent with the median Mauna 
Kea turbulence. The cost of such a system is significant and is only used for testing purposes. The NFIRAOS team has identified several reasonable mitigation strategies to compensate for the loss of such a system, including but not limited to i) exercising NFIRAOS in LTAO mode where the 2 two DMs are used to generate turbulence but only DM0 is used to correct it, ii) using the turbulence generator prototype screen during the integration and test phase, and iii) creating turbulence with hot air when testing the system at room temperature. Removing the turbulence generator has additional benefits such as reducing the mass and freeing the space to include a rotating pupil mask, which may be required for calibrations.

- The source simulator system is quite complex and includes the 3 main components: a focal plane mask, 16 diffraction limited, natural guide star sources arranged in 4 rings with fixed and adjustable brightness, and 6 laser guide star sources with adjustable brightness and adjustable sodium layer range. The final design of such system has been successfully completed, but again the cost to build and test such a system is significant. Different options are considered to reduce the cost of this system including simpler tests, giving up on the natural guide sources, giving up on their variability only, etc.... These tradeoffs are being finalized.

- Recently, the NFIRAOS team has recognized the benefit of adding a retractable and rotating pupil mask in the NFIRAOS common path. This mask would simulate the TMT exit pupil or NFIRAOS entrance pupil, including the serrated pupil edge and the secondary mirror support structures. Such a pupil mask was originally included in the NFIRAOS Science Calibration Unit (NSCU), which is located in front of the NFIRAOS entrance window. Including the rotating pupil mask within NFIRAOS (in the space vacated by the turbulence generator) seems to be a more cost effective and technically better solution. This tradeoff is still on-going.
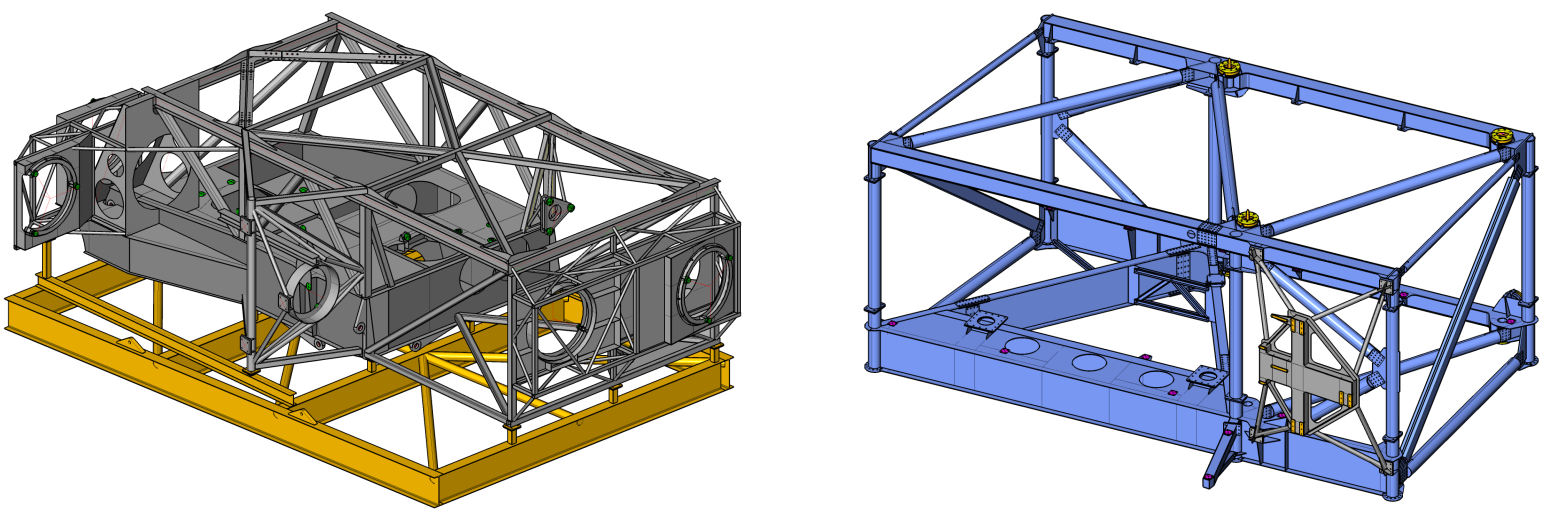

Figure 3: Final Design of the NFIRAOS optical table (left) and instrument support tower (right) by Sightline Engineering.
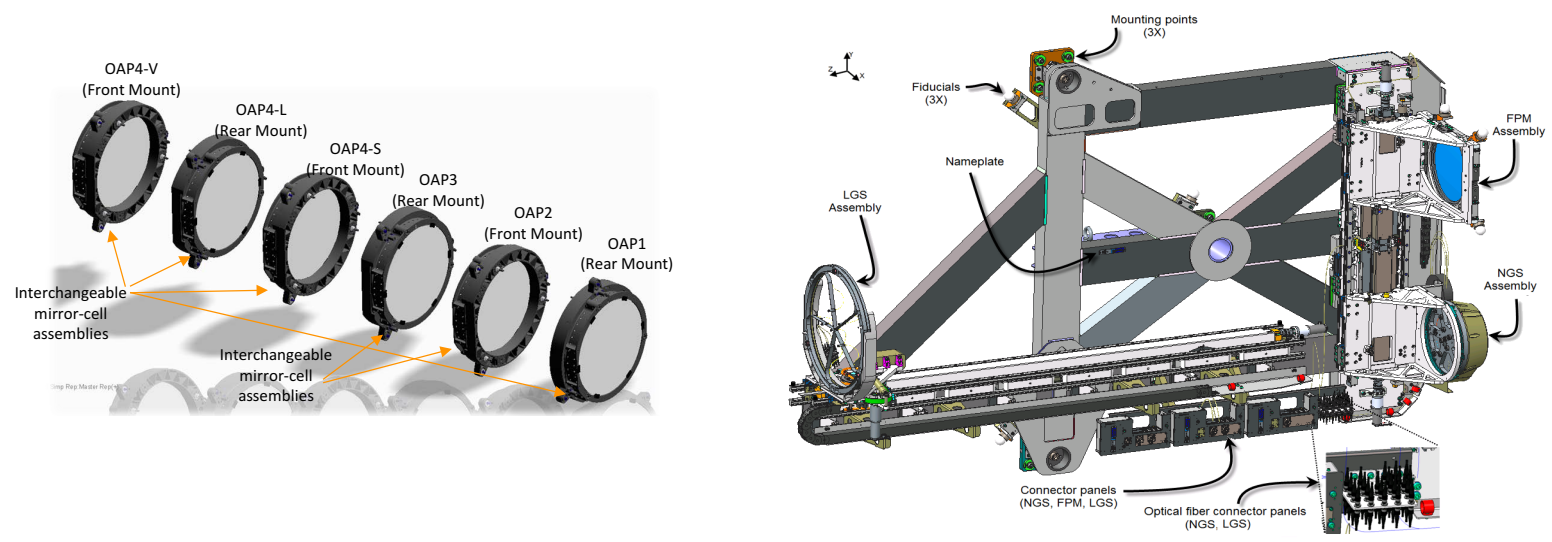

Figure 4: Final Design of the NFIRAOS off axis parabolas (left) by ABB and Source Simulator (right) by INO including the LGS sources on a stage, the NGS sources and the focal plane mask. 


\subsection{Laser Guide Star Facility (LGSF)}

The Institute of Optics and Electronics (IOE) team in Chengdu, China, is developing the LGSF preliminary design with the support of the TMT AO team. Since the AO4ELT5, major modifications of the design have been done ${ }^{[5]}$.

- Reduction of the largest asterism: the LGSF can generate 4 different asterisms on the sky for NFIRAOS and for 3 next generation AO instruments. The largest asterism consists of 4 LGS located at the corners of a 9 arcmin by 4 arcmin rectangle, offset by 4.8 arcmin along the short edge. This asterism is dedicated to wide field optical spectroscopy using Ground Layer AO (GLAO). Based on recent simulations to assess the performance of an adaptive secondary system for TMT, it was found that this asterism should be reduced to a 6 arcmin by 4 arcmin rectangle to be optimal. Reducing this asterism allows to reduce the size of the asterism generator. To further reduce the size of the optics between the asterism generator and the laser launch telescope, the offset of the asterism is now performed by the launch telescope.

- The LGSF top end design was simplified to reduce complexity and improve access for maintenance:

- The diagnostic alignment cameras were replaced with a diagnostic wavefront sensor and several prealignment cameras along the optical path. The purpose of the diagnostic wavefront sensors is to measure the pointing and centering errors of all the laser beams, as well as their beam quality. These errors are corrected using the asterism generator positioning mirrors. The pre-alignment cameras are used to do a rough alignment of the LGSF optical path.

- The complexity of the top end was reduced by removing the redundancy of some of the positioning actuators within the asterism generator. The asterism generator is used to transform the array of laser beams into the proper asterism.

- The previous radial design of the asterism generator was abandoned and replaced by a bench design to improve access to its components (see Figure 6).

- Finally, the entire layout of the LGSF top end was modified to provide access to the back of the telescope secondary mirror.

- The design of the Laser Launch Telescope (LLT) was updated from an off axis reflective design to a refractive design to relax the mechanical tolerances without impacting the performances.
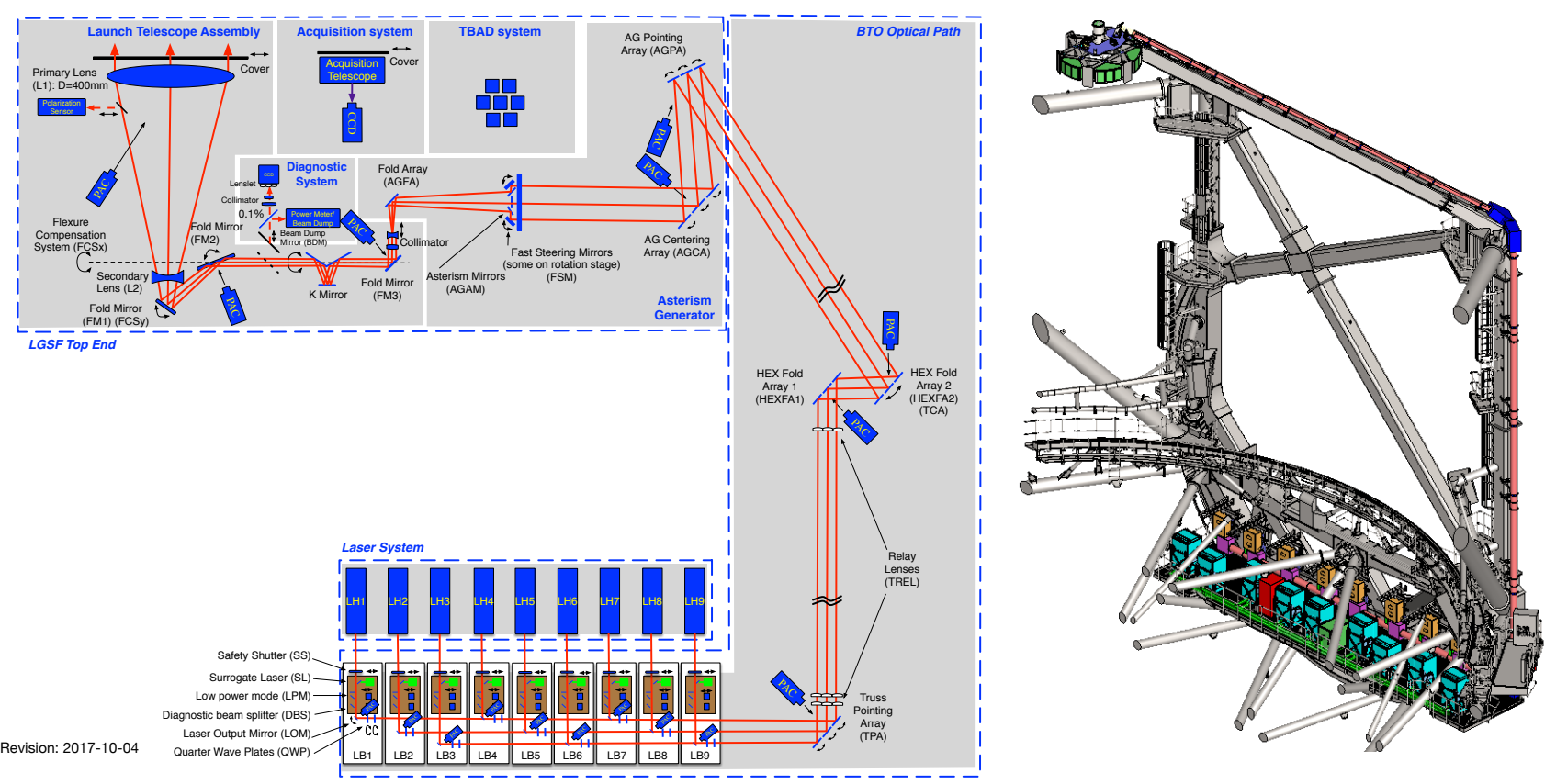

Figure 5: Updated LGSF layout (left) and detailed interface of the LGSF with the telescope structure (right). 
The overall layout has been updated and the detailed interface to the telescope structure has been updated and finalized (see Figure 5). The LGSF system extends from the laser platform to the telescope top end, and has many complex structural, mechanical, access and handling, services and utilities, and safety interfaces to the telescope structure. A total of 20 space envelopes and attachment drawings have been developed.
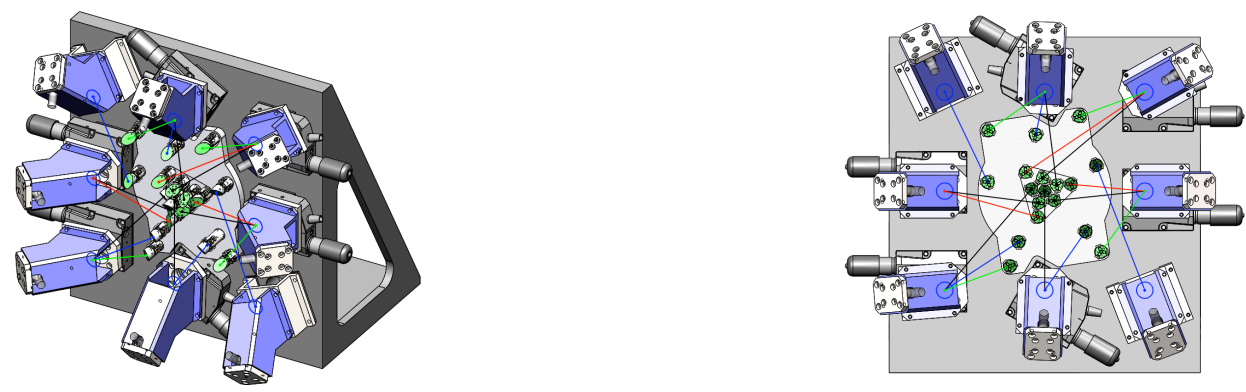

Figure 6: Updated design of the LGSF asterism generator.

\subsection{Adaptive Optics Executive Software}

The AO Executive Software is composed of three sub-systems: i) the AO Sequencer, which coordinates the actions of the AO systems, ii) the Reconstructor Parameter Generator (RPG), which computes the AO parameters needed by the NFIRAOS Real Time Controller, and, iii) the Point Spread Function (PSF) Reconstructor, which post-processes the AOcorrected PSF from the NFIRAOS WFS and DM telemetry data.

A fully automated AO Sequencer is required due to the complexity of the interactions between AO systems and the TMT requirement for high observing efficiency. This software system will be central to performing AO-assisted observations. It will perform the control and monitoring of the NFIRAOS sub-systems, LGSF sub-systems, On-Instrument WFSs and On Detector Guide Windows of the NFIRAOS instruments. The AO Sequencer components will be dynamically created and composed to execute the startup, test, calibration, observation and shutdown sequences. The observation sequences also include sub-sequences for acquisition, nodding and dithering, non-sideral object tracking, calibration and recovery after a laser safety event or $\mathrm{AO} /$ telescope fault.

Since the AO4ELT4, the TMT AO team has focused on the following activities:

- Successfully completing the preliminary design work of the Reconstructor Parameter Generator (RPG). The most demanding task of the RPG is to compute and transfer the high order control matrix for the LGS reconstruction to the NFIRAOS real time controller every 10 seconds as the atmospheric turbulence parameters change. The high order control matrix is computed using Fourier Domain Pre-Conditioned Gradient (FDPCG) and requires high performance server based computer using a combination of CPUs and GPUs. Interface to the RTC is done via a dedicated $10 \mathrm{GbE}$ link.

- Detailing the different sequences and in particular the acquisition sequences with goal to optimize and verify timing requirements ${ }^{[8],[10]}$.

- Continuing to develop the PSF Reconstruction algorithms ${ }^{[11]}$. Since the AO4ELT4, the work has focused on developing the NGS AO algorithms first. A total of 4 algorithms have been tested on simulated telemetry data and actual laboratory telemetry data. The actuator telemetry based algorithm seems to be the most promising and meets TMT requirements. Such algorithm can naturally be extended to tomographic PSF Reconstruction.

\section{FIRST LIGHT AO COMPONENT DEVELOPMENT}

\subsection{Deformable Mirrors}

The requirements for the NFIRAOS DMs includes two mirrors of order $63 \times 63$ and $76 \times 76$ with a $5 \mathrm{~mm}$ inter-actuator spacing, $10 \mu \mathrm{m}$ of stroke after flattening, $15 \%$ hysteresis, less than $2 \%$ per time decade creep and an operating temperature of $-30^{\circ} \mathrm{C}$. 
TMT is currently funding two competitive studies with AOA Xinetics and CILAS to develop the final designs of the NFIRAOS DMs, and to fabricate new prototypes to be delivered to the NRC Herzberg for testing during the spring of 2018. Both contracts are progressing well at this point.

\subsection{High-order LGS and NGS Wavefront Sensing}

For the six NFIRAOS 60x60 LGS WFS, TMT will use the polar coordinate CCDs designed specifically for elongated laser guide star images. The polar coordinate CCD reduces the pixel count and total readout rate by roughly a factor of three in comparison to a standard CCD with a conventional rectangular geometry. The requirements for such a detector for TMT include sub-aperture sizes varying from $6 \times 6$ pixels at the center of the array to $6 \times 15$ pixels at the edge, a quantum efficiency of $90 \%$, and $\sim 3$ electrons read noise at a frame rate of $800 \mathrm{~Hz}$.

A one-quadrant prototype of the TMT polar coordinate CCD was successfully designed and fabricated in a wafer run at MIT/LL funded by the TMT, Keck and USAF Research Laboratory. Based on this success, TMT has launched the design and development of the full-scale polar detector at MIT/LL (designated as the CCID87). The design of the fullscale detector is based on the quadrant prototype design. The quadrant design is mirrored twice to fill a 360 arc, and a third metal layer is added to avoid gaps between the quadrants. A first lot of sixteen, $200 \mathrm{~mm}$ wafers of $14 \mathrm{CCDs}$ each, is now being fabricated, with the first eight wafers fully processed and tested through front illumination and the second set of eight wafers fully processed and being tested through front illumination. In parallel, four of the eight first wafers have been fully processed through back illumination and are being tested.
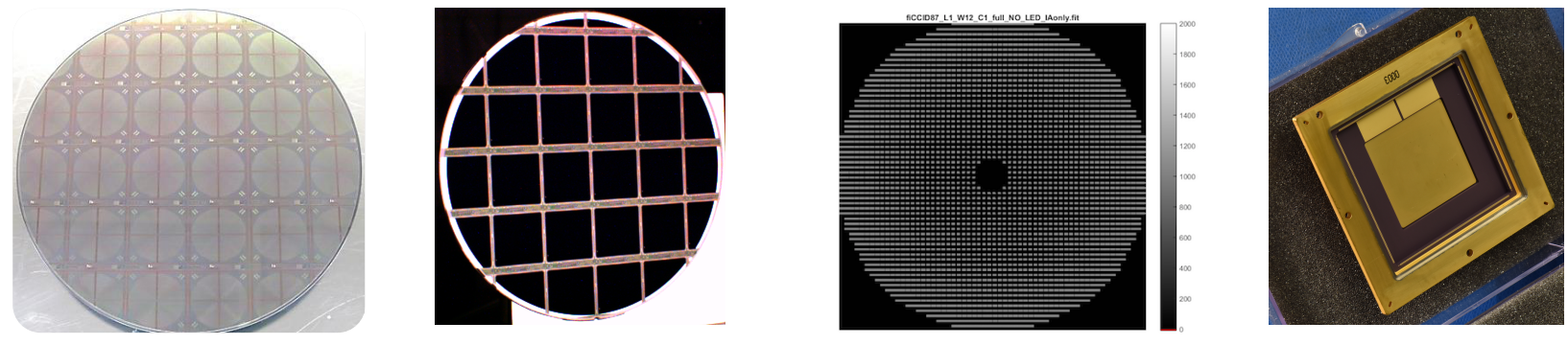

Figure 7: From left to right: 1) Finished front illuminated CCID87 wafer, 2) Finished back illuminated CCID87 wafer, 3 ) CCID87 front side dark image at $20^{\circ} \mathrm{C}$, 4) 480 pin CCID 87 package developed by Kyocera.

Fully functional science grade devices have been obtained after front illumination with the first set of 8 wafers, although the yield has been lower than expected. The design and the front illumination process have been updated to improve the yield and will be tested with the second set of 8 wafers. Preliminary results show no further degradation following the back-illumination process ${ }^{[7]}$.

For the NFIRAOS NGS Pyramid WFS, TMT will use the 256x256 CCD array (designated as the CCID74) developed by MIT/LL as part of the polar coordinate prototype detector effort. This CCD has been successfully tested and achieves the requirements of $80 \%$ quantum efficiency and $\sim 1$ electron read noise at a $100 \mathrm{~Hz}$ frame rate. Two science grade devices will be packaged for TMT using the same detector package developed for the polar coordinate detector. The LGS and NGS WFS cameras for NFIRAOS will also be based on a common design.

The preliminary design of the NFIRAOS LGS and NGS WFS cameras and readout electronics has been contracted to Astronomical Research Cameras (ARC) in San Diego, with Quartus Engineering as a sub-contractor for the optomechanical design. The main performance requirements of the readout electronics and camera are summarized here:

- Fast pixel data rates for the readout electronics: 128 parallel outputs at 3.5 million pixels per second per output, with a total of $\sim 205,000$ pixels read out in $500 \mu \mathrm{sec}$ for the polar coordinate detector.

- Low noise for the readout electronics $(<3$ electrons for the polar coordinate detector and $<1$ electron for the NGS detector), requiring the electronics to be packaged close to the detector to avoid long and large interconnections.

- Small volume constraints and less than $0.5 \mathrm{~W}$ heat dissipation per LGS and NGS camera, as they are located within the $-30^{\circ} \mathrm{C}$ NFIRAOS cold enclosure. 

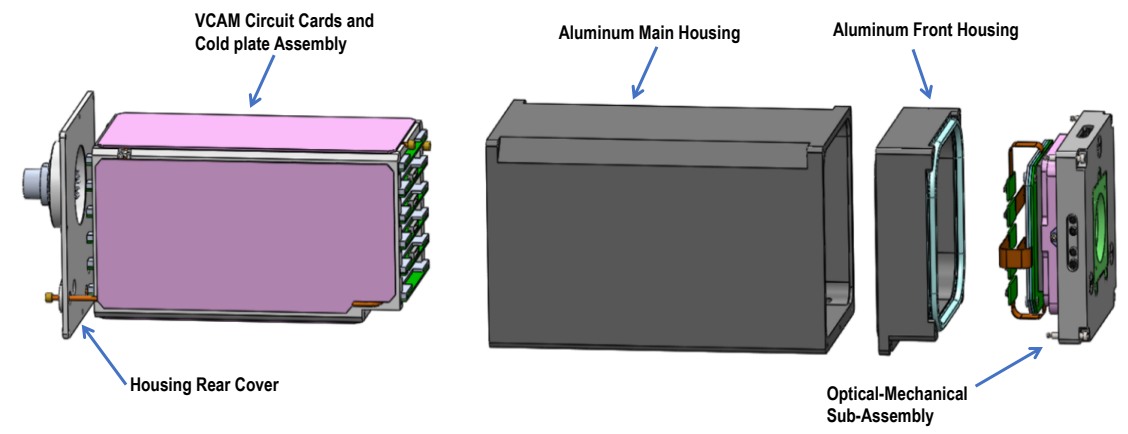

Figure 8: CAD image of the NFIRAOS LGS and NGS visible WFS cameras, including the focal plane readout electronics.

The ARC/Quartus team has successfully completed the preliminary design of the cameras and readout electronics. ARC has also developed a 32 channel sub-scale readout electronics prototype and is testing this prototype with an engineering grade NGS WFS 256x256 detector ${ }^{[7]}$. First images have recently been obtained with the readout electronics prototype and the engineering grade detector at room temperature and without optimization (see Figure 9).
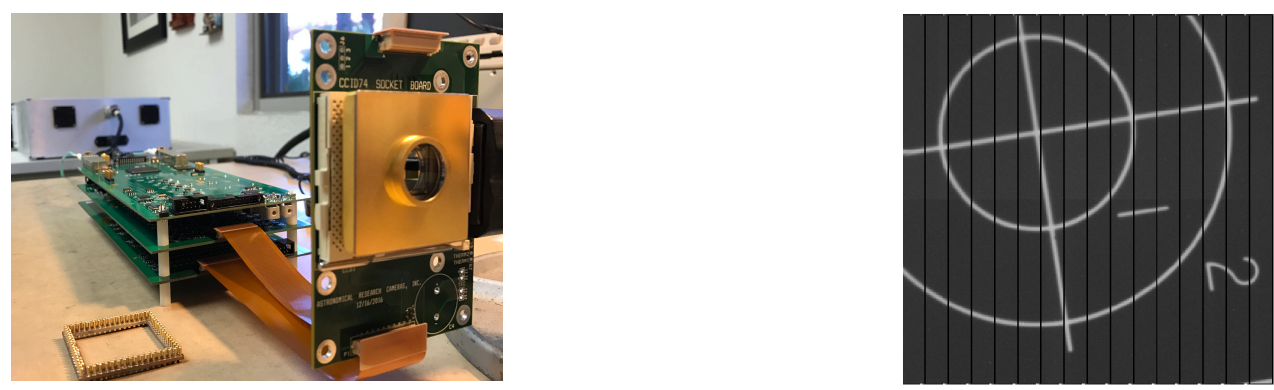

Figure 9: Left: ARC readout electronics prototype. Right: Early test image from VCAM using an engineering grade CCID74 (the vertical black lines are deliberate to allow easier identification of individual video channels)

\subsection{Low-order On-Instrument NGS Wavefront Sensing}

TMT presently intends to use Teledyne Hawaii-2RG detectors with $1 \mathrm{Kx} 1 \mathrm{~K}$ science grade sub-arrays for the low-order On-Instrument WFS detectors in the NFIRAOS instruments. These detectors provide a large enough field of view for initial acquisition, very good quantum efficiency in the $\mathrm{J}, \mathrm{H}$ and $\mathrm{K}$ bands, and low read noise for small sub-arrays at frame rates of $10-400 \mathrm{~Hz}$. An alternative detector under consideration is the Selex APD. This detector appears to provide superior read noise performance on larger sub-arrays at the required frame rate, although the current device with $256 \mathrm{x}$ 320 pixels is still too small for initial guide star acquisition.

The use of on-instrument wavefront sensors has now been augmented with the definition of an on-detector guide window capability for the IRIS imager. Up to four guide windows can be used for either fast tip/tilt sensing or lowerbandwidth "truth" sensing of slowly varying alignment errors, depending upon the brightness of the guide stars. The use of the OIWFS and the ODGW will be fully flexible and interchangeable to the NFIRAOS RTC, apart from the requirement to have at least one OIWFS for tip/tilt/focus sensing.

\subsection{Real Time Controller}

The requirements for the NFIRAOS Real Time Controller include real time pixel processing for the high-order LGS and low-order On-Instrument NGS wavefront sensors, tomographic wavefront reconstruction and calculation of the wavefront corrector actuator commands (requiring to solve a $35 \mathrm{k}$ x $8 \mathrm{k}$ control problem at $800 \mathrm{~Hz}$ ), and real-time optimization of the algorithms for these processes as atmospheric and observing conditions change. The RTC also acquires wavefront corrector and wavefront sensor telemetry data in order to estimate the science Point Spread Function (PSF) for image post-processing. 
The NRC-Herzberg team is completing the final design of the NFIRAOS Real Time Controller. The system architecture is based upon commercial CPU server hardware with a classical Matrix Vector Multiply (MVM) control algorithm (see Figure 10). The NFIRAOS Real Time Controller resides in the computer room and connection to and from the AO components is done via dedicated $10 / 40 \mathrm{~Gb}$ Ethernet switches.

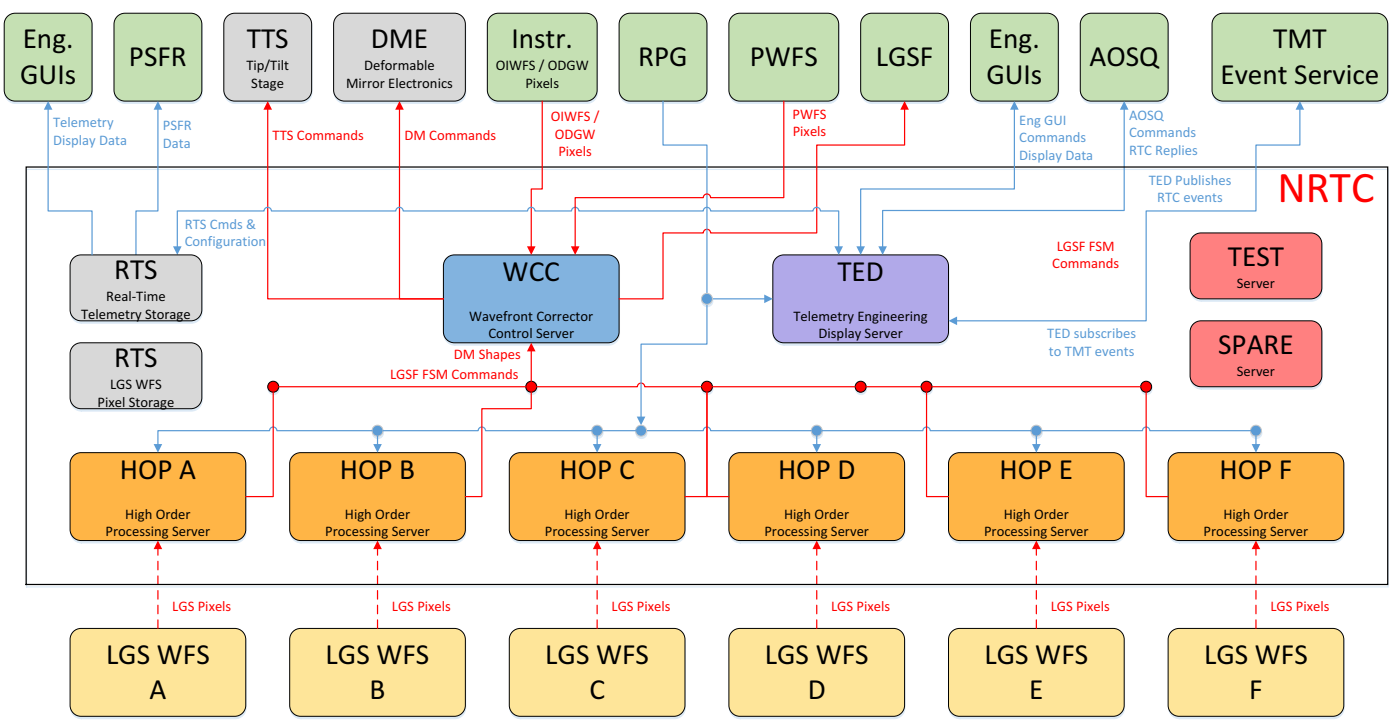

Figure 10: The RTC includes a total of ten servers with one server per LGS WFS, one server dedicated to wavefront corrector control, one server dedicated to telemetry engineering display, a test server and a spare server. Each High Order Processing (HOP) server currently includes four high end Intel Xeon CPUs. The connection between the servers, to and from the AO components and other systems is performed through a 10/40Gb Ethernet switch with 32 ports.

\subsection{Lasers}

The TMT first light Laser Guide Star Facility will utilize six $25 \mathrm{~W}$ sodium guide star lasers $\left(20 \mathrm{~W}\right.$ with the $\mathrm{D}_{2} \mathrm{a} / \mathrm{D}_{2} \mathrm{~b}$ repumping option) with high beam quality, high coupling efficiency, high reliability, and a design compatible with a variable gravity vector orientation and the harsh environment of an astronomical observatory.

TMT intends to use Toptica/MPBC lasers. The Toptica/MPBC laser is a Raman Fiber laser which produces the $589 \mathrm{~nm}$ beam using second harmonic generation (SHG) conversion of a $1178 \mathrm{~nm}$ laser. The Toptica/MPBC laser meets the TMT performance and engineering requirements and has been successfully commissioned at the ESO VLT with the 4LGSF system, and at Keck with the Keck II NGL system.

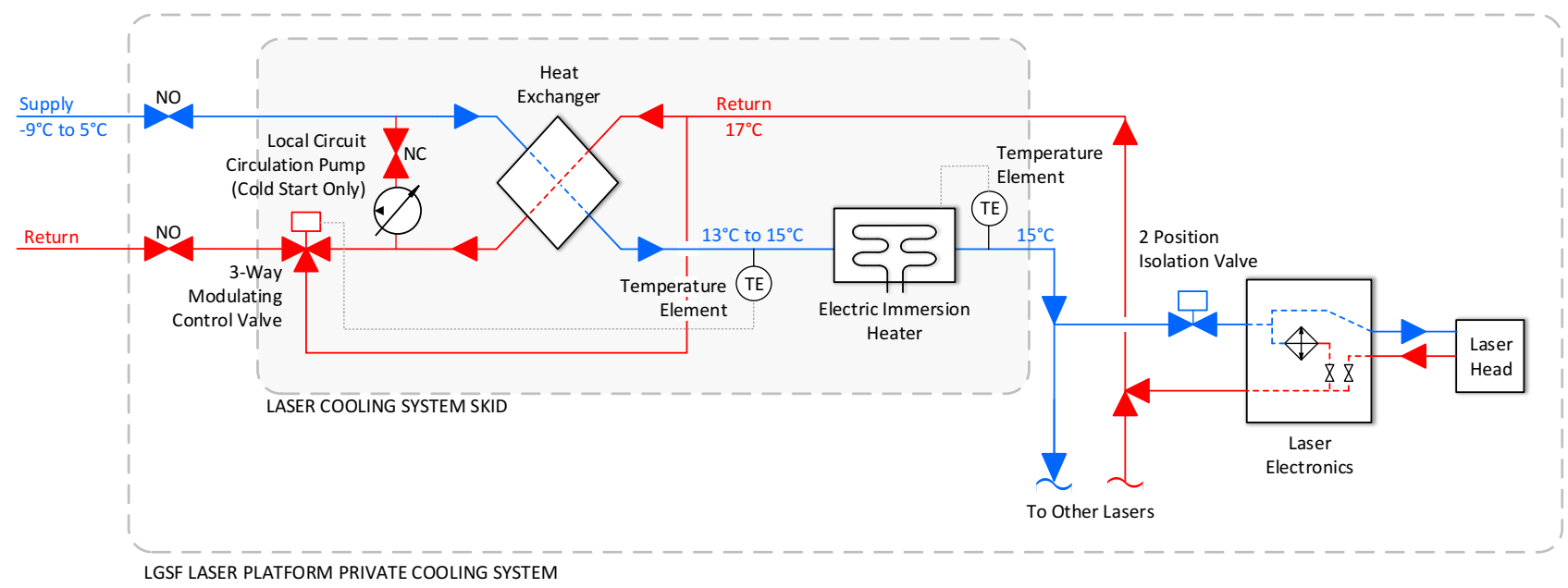

Figure 11: The Laser Cooling System schematic 
Since the last AO4ELT4 conference, TMT has developed the interfaces to this laser and a cooling system concept for up to eight Toptica/MPBC lasers. The cooling system will be mounted on the laser platform and will include a heat exchanger and a heater, which will be used to warm the laser electronics during the day and maintain the proper operating temperature during the night using the waste heat generated by the lasers while they are lasing (see Figure 11).

\section{AO SYSTEM ENGINEERING, MODELING AND PERFORMANCE ANALYSIS}

Several AO modeling and analysis activities have been conducted at TMT, in particular in the areas of AO requirements definition, wavefront error budget development, pyramid WFS performance modeling for the NGS AO mode and truth wavefront sensing, and adaptive secondary mirror performance for TMT ${ }^{[1],[6],[9],[12],[13]}$.

\subsection{AO Budget Updates}

The NFIRAOS wavefront error budget has been updated for the LGS MCAO operating mode to distinguish between requirements and current best estimates, to incorporate design changes and new analysis results, and to better identify traceability to/from other requirements. In particular, the LGS MCAO current best estimate has been updated i) to further detail the NFIRAOS error terms (such as optical alignment and polishing terms), ii) to include the IRIS wavefront error budget, iii) to include the telescope mechanical vibration budget, and iv) to include the LGSF contribution to the LGS MCAO wavefront error budget and applicable error terms from the AO components, such as the DM dynamic effects. A total of 72 terms for the LGS MCAO wavefront error budget have been assessed through simulations and traced up to requirements ${ }^{[4]}$. The LGS MCAO wavefront error budget top-level budget is given in Table 1. Similar work has been performed to update and detail the NGS AO wavefront error budget. The LGS MCAO and NGS AO expected Strehl Ratio performance are provided in Table 2 and Table 3.

Table 1: Updated top-level LGS MCAO performance wavefront error for NFIRAOS + IRIS.

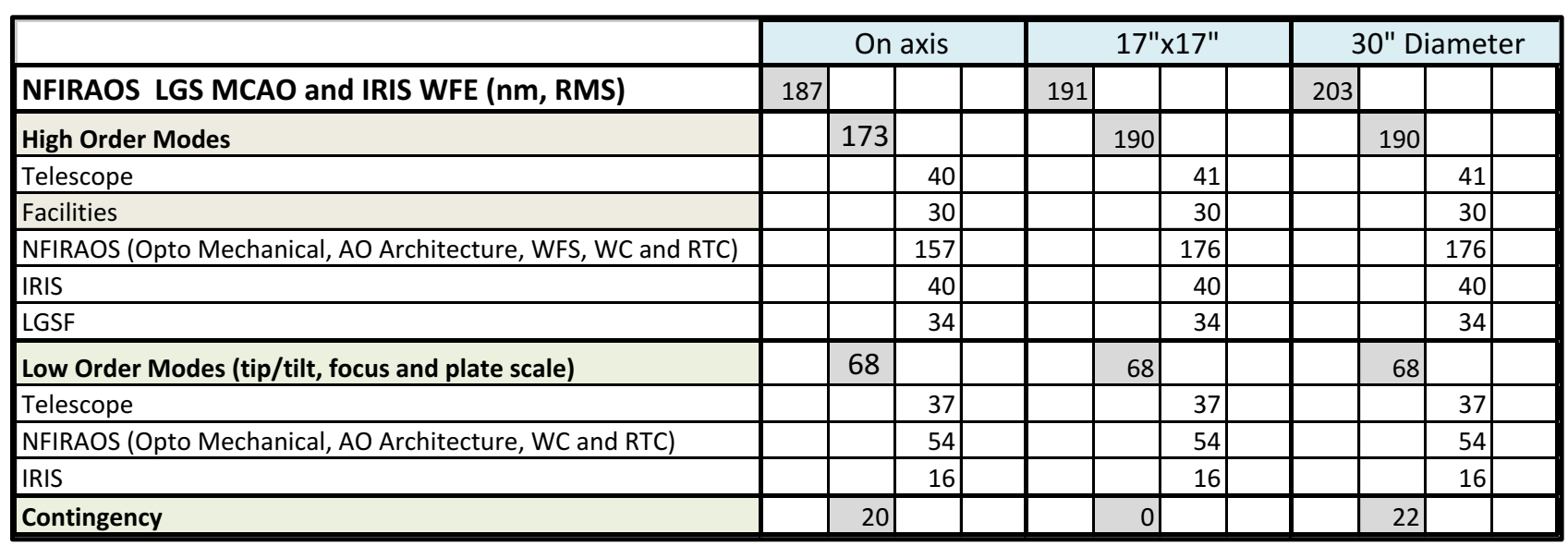

Table 2: LGS MCAO Strehl Ratio estimates for NFIRAOS + IRIS

\begin{tabular}{|l|c|c|c|}
\hline LGS MCAO & On axis & $17 " x 17 "$ & $30 "$ Diameter \\
\hline SR(J), \% & $41.3 \%$ & $39.8 \%$ & $35.2 \%$ \\
\hline SR(H), \% & $60.2 \%$ & $58.9 \%$ & $55.0 \%$ \\
\hline SR(K), \% & $74.7 \%$ & $73.8 \%$ & $70.9 \%$ \\
\hline
\end{tabular}

Table 3: NGS AO Strehl Ratio estimates for NFIRAOS + IRIS

\begin{tabular}{|l|c|c|}
\hline NGS AO & $m R=8$ guide star & $m R=12$ guide star \\
\hline SR(J), \% & $51.0 \%$ & $42.1 \%$ \\
\hline SR(H), \% & $68.0 \%$ & $60.9 \%$ \\
\hline SR(K), \% & $80.1 \%$ & $75.2 \%$ \\
\hline
\end{tabular}




\subsection{Adaptive Secondary Mirror Performance Assessment}

A new round of modeling has been conducted to assess the performance of an adaptive secondary mirror (AM2) for TMT. Such a system would i) enable ground layer AO correction for the TMT seeing limited and large field of view instrument, ii) reduce emissivity for a mid-infrared instrument (between 3 and 25 microns) by removing an AO relay, and iii) eliminate the need for additional large stroke, low-order woofer deformable mirrors for the next generation of TMT instruments requiring Extreme AO, Multi-Object AO or for an upgrade of NFIRAOS to a 120x120 correction order system. The goals of the study were to i) quantify the impact of having the TMT adaptive secondary mirror at a negative conjugation (TMT uses a Ritchey Chrétien design and the secondary mirror is conjugated at $-280 \mathrm{~m}$ ), ii) optimize the LGS asterism for a Ground Layer AO (GLAO) instrument, iii) define the required order of correction of the TMT adaptive secondary mirror for the different TMT AO systems and iv) compare the performance of such a system for Mauna Kea (MK) or for the Observatorio del Roque de los Muchachos (ORM). The results show that:

- A 30x30 order correction is sufficient for various turbulence profiles for GLAO correction as shown in Figure 12 (left figure). Such order of correction is also sufficient for woofer operations or for mid infrared AO correction. Instruments with a small field of view would benefit from a higher order system, however.

- A -280m conjugation has only minimal performance impacts compared to a 0m conjugation as shown in Figure 12 (middle figure). Between 10 and 30\% improvement is obtained in FWHM for a GLAO correction using a $30 \times 30$ order AM2 and for medium seeing. The small penalty is due to anisoplanatism coming from all layers.

- An optimized GLAO asterism (6 arcmin to 4 arcmin rectangle) has been defined for the TMT seeing limited instrument as shown in Figure 12 (right figure). Impact due to conjugation increases and correction degrades with larger correction field of view

- Finally, performance of a TMT AM2 are noticeably better for the MK site than for the ORM site, because the current data shows that ORM has less ground layer turbulence than MK.
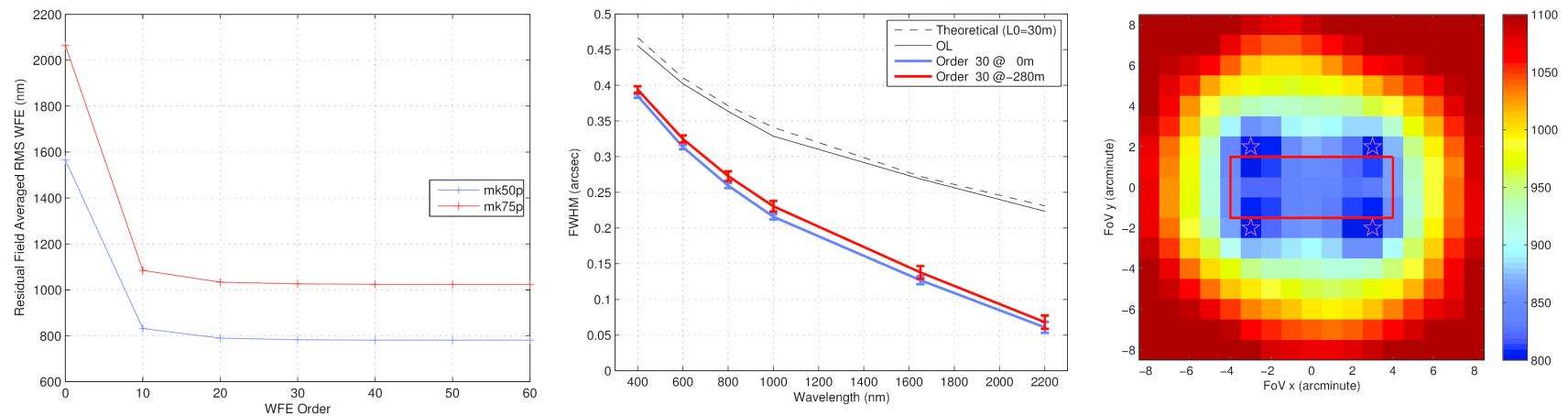

Figure 12: AM2 performance Assessment. Left: AM2 order of correction versus GLAO performance. A 30x30 order of correction is sufficient for GLAO. Middle: GLAO performance versus conjugation for a 30x30 order AM2. Impact of -280m conjugation versus $0 \mathrm{~m}$ is small. Right: For Medium Mauna Kea turbulence profile, a rectangular 6'x4' asterism gives optimum performance.

\section{SUMMARY}

Significant progress has been accomplished in the design, prototyping and modeling of the TMT first light AO systems and AO components. Design and prototyping activities have taken place at the NRC-Herzberg Institute in Canada, at IOE in China, and at TMT in Pasadena to advance the NFIRAOS, LGSF and AOESW design effort. Progress in AO components development is also continuing for i) the final design and prototyping of the deformable mirrors, ii) the fabrication and test of the full scale polar coordinate detector for the LGS WFS, and the readout electronics prototyping testing of the LGS and NGS visible cameras, and iii) the final design of the NFIRAOS RTC. Extensive work is also being performed in the area of AO system engineering and modeling to better define and consolidate our design and interface requirements. 


\section{ACKNOWLEDGMENTS}

This paper is a summary of the contributions of numerous TMT staff members, TMT partners and TMT suppliers including: Lianqi Wang, Luc Gilles, Melissa Trubey (TMT AO Group), Brent Ellerbroek, Sean Adkins (Consultants), Gelys Trancho, Hugh Thompson, Tony Travouillon, Angel Otárola (TMT), Glen Herriot, David Andersen, Jenny Atwood, Peter Byrnes, Kris Caputa, Jeff Crane, Adam Densmore, Jennifer Dunn, Joeleff Fitzsimmons, Brian Hoff, Dan Kerley, Olivier Lardiere, Malcom Smith, Jonathan Stocks, Jean-Pierre Véran (NRC-Herzberg), Kai Wei, Muwen Fan, Changchun Jiang, Min Li, Xiqi Li, Daoman Riu, Jinlong Tang, (IOE), Aaron Castillo, Jeff Cavaco, Audrey Brooks, John Vayda (AOA Xinetics), Hubert Pagès, Jean-Christophe Sinquin, Aurelien Moreau, Gabrielle Dutey, Stephane Vaillant (CILAS), Bob Leach (ARC), Kyle Laudner (Quartus), Mark Fraser (M3 Engineering), James Gregory, Bradley Felton, Kevin Ryu, Michael Brattain, Daniel O’Mara (MIT/LL), Wilhelm Kaenders, Bernhard Ernstberger (Toptica)

The TMT Project gratefully acknowledges the support of the TMT collaborating institutions. They are the Association of Canadian Universities for Research in Astronomy (ACURA), the California Institute of Technology, the University of California, the National Astronomical Observatory of Japan, the National Astronomical Observatories of China and their consortium partners, and the Department of Science and Technology of India and their supported institutes. This work was supported as well by the Gordon and Betty Moore Foundation, the Canada Foundation for Innovation, the Ontario Ministry of Research and Innovation, the National Research Council of Canada, the Natural Sciences and Engineering Research Council of Canada, the British Columbia Knowledge Development Fund, the Association of Universities for Research in Astronomy (AURA), the U.S. National Science Foundation, and the National Institutes of Natural Sciences of Japan.

\section{REFERENCES}

[1] Herriot G., et al., "Point Source Sensitivity, Pupil alignment, Calibration and Control for TMT-NFIRAOS-IRIS," AO4ELT5 Proceedings, (2017).

[2] Wright S., et al., "Science Development for IRIS (Infrared Imaging Spectrograph) on TMT," AO4ELT5 Proceedings, (2017).

[3] Herriot G., et al., "NFIRAOS," AO4ELT5 Proceedings, (2017).

[4] Hardie, K., et al., "Thirty Thirty Meter Telescope Adaptive Optics System Error Budgets and Requirements Traceability," AO4ELT5 Proceedings, (2017).

[5] Trubey, M, et al., "TMT Laser Guide Star Facility Update," AO4ELT5 Proceedings, (2017).

[6] Suzuki R., et al., "Instrument requirement for precision relative astrometry using TMT/IRIS," AO4ELT5 Proceedings, (2017).

[7] Adkins, S., et al., "Laser guide star optimized wavefront sensor system for the Thirty Meter Telescope," AO4ELT5 Proceedings, (2017).

[8] Trancho G., et al. "Analyzing the Operational Behavior of NFIRAOS LGS MCAO Acquisition on the Thirty Meter Telescope using SysML," AO4ELT5 Proceedings, (2017).

[9] Ellerbroek B. "Roof- and Pyramid Wavefront Sensing with Extended Sources," AO4ELT5 Proceedings, (2017).

[10] Andersen D., et al., "Acquisiton with the TMT IRIS On-Instrument Wavefront Sensor System," AO4ELT5 Proceedings, (2017).

[11] Gilles L., et al., "PSF reconstruction analysis, modeling, simulations, and tests on the HeNOS bench at NRCHerzberg," AO4ELT5 Proceedings, (2017).

[12] Wang, L., et al, "Pyramid WFS Tolerance Study for NFIRAOS NGS AO,” AO4ELT5 Proceedings, (2017).

[13] Véran, J.P., et al., "Telescope pupil tracking using a Pyramid WFS,” AO4ELT5 Proceedings, (2017). 